\title{
Linx
}

Revue des linguistes de l'université Paris X Nanterre

64-65 | 2011

Les genres de discours vus par la grammaire

\section{Présentation : du genre vers la grammaire}

\section{Mustapha Krazem}

\section{OpenEdition}

Journals

Édition électronique

URL : http://journals.openedition.org/linx/1393

DOI : 10.4000/linx.1393

ISSN : 2118-9692

\section{Éditeur}

Presses universitaires de Paris Nanterre

\section{Édition imprimée}

Date de publication : 1 juillet 2011

Pagination : 7-14

ISSN : 0246-8743

\section{Référence électronique}

Mustapha Krazem, «Présentation : du genre vers la grammaire », Linx [En ligne], 64-65 | 2011, mis en ligne le 01 juillet 2014, consulté le 22 septembre 2020. URL : http://journals.openedition.org/linx/1393 ; DOI : https://doi.org/10.4000/linx.1393 


\title{
Présentation : du genre vers la grammaire
}

\author{
Mustapha Krazem
}

La recherche des relations entre genre de discours et grammaire était, jusqu'à récemment, plutôt réduite. Du moins, si on prend le point de vue des chercheurs spécialisés dans l'identification et la description des faits internes aux systèmes linguistiques. De leur côté, les linguistes (principalement issus de l'analyse du discours et de la linguistique textuelle) qui se sont intéressés aux genres - et on sait les nombreux travaux, colloques, projets scientifiques consacrés à cette question - ont immédiatement perçu la place décisive des faits de langue dans la description des genres. Et pour cause : Bakhtine, dont les écrits ont largement inspiré une partie de la communauté linguistique, affirmait que la langue s'apprenait en même temps que les genres de discours, ce qui a été largement repris depuis, totalement ou partiellement (Rastier, 2001, Dolinine, 1999). Jean-Jacques Richer montrera dans ce numéro l'intérêt d'intégrer les genres dans la didactique du F.L.E.

La recherche de marqueurs linguistiques caractérisant les genres (par exemple les genres procéduraux, les genres de la presse etc.) a ainsi été à l'origine de nombreux travaux. Nous ne nous lancerons pas ici dans un récapitulatif exhaustif ou un état des lieux de ces recherches. Nous préférons insister sur le fait que les linguistes qui ont bénéficié de l'impulsion bakhtinienne admettent volontiers que la notion de genres de discours dépourvue de son appareillage grammatical se réduit à peu de choses et relève davantage de la science de la communication, de la sociologie, voire de l'économie que de la linguistique. 
Pourtant, l'impulsion bakhtinienne n'a guère eu d'échos chez les grammairiens professionnels, surtout les syntacticiens. Jusqu'à une période récente, les genres de discours, sans nécessairement d'ailleurs apparaittre sous cette appellation, n'étaient sollicités que pour limiter ou appuyer une règle. Par exemple, l'inversion simple verbesujet qui est observable dans les didascalies. Nous nous attardons sur ce point dans (Despierres/Krazem, 2012b) à propos des grammaires générales récentes. Le tableau ci-dessous confirme que celles-ci prennent en compte les genres de discours pour illustrer des points de langue isolés. Plusieurs faits dont on sait qu'ils sont sensibles à leur immersion générique avaient été ciblés : les phrases averbales, l'inversion du sujet nominal, l'infinitif de prescription et le présent de l'indicatif. Nous constatons alors fréquemment que les exemples font référence explicitement (sans italiques) ou implicitement (en italiques) à des genres.

(1) Insertion des genres de discours dans quelques grammaires

\begin{tabular}{|c|c|c|c|c|}
\hline & $\begin{array}{l}\text { Phrases } \\
\text { nominales }\end{array}$ & $\begin{array}{l}\text { Inversion du } \\
\text { sujet nominal }\end{array}$ & Infinitif & Présent indicatif \\
\hline Bonnard & Journalisme & $\begin{array}{l}\text { txt administratif } \\
\text { français littéraire }\end{array}$ & entrer sans frapper & Sportif \\
\hline Sancier-Chateau & & $\begin{array}{l}\text { Ici repose Gustave } \\
\text { Flaubert }\end{array}$ & $\begin{array}{l}\text { Ne pas fumer } \\
\text { Guides, } \\
\text { recettes, } \\
\text { prescriptions } \\
\text { officielles }\end{array}$ & $\begin{array}{l}\text { Énoncés } \\
\text { définitoires, } \\
\text { maximes, } \\
\text { sentences, } \\
\text { présent } \\
\text { historique }\end{array}$ \\
\hline Le Goffic & $\begin{array}{l}\text { Stationnement } \\
\text { interdit } \\
\text { Indications } \\
\text { scéniques, } \\
\text { titres de } \\
\text { journaux, } \\
\text { dictons }\end{array}$ & $\begin{array}{l}\text { Ici est né le sculpteur } \\
X \\
\text { Indication } \\
\text { scénique, } \\
\text { style } \\
\text { télégraphique: } \\
\text { langage } \\
\text { administratif }\end{array}$ & $\begin{array}{l}\text { Ne pas fumer } \\
\text { Ne pas dépasser la } \\
\text { dose prescrite } \\
\text { Laisser mijoter à } \\
\text { feu doux }\end{array}$ & \\
\hline
\end{tabular}

Mais, le plus souvent, les études syntaxiques ignorent les genres de discours, bien que Dominique Klinger, dans ce numéro, montre que ce souci existait déjà chez le linguiste Henri Frei. Souvent, les genres du discours sont/(étaient) relégués dans des espaces mal définis plus ou moins théorisés : contexte/hors contexte, performance/ compétence ou encore faits de langue/faits de discours.

Les linguistes qui se sont intéressés à la sémantique verbale font exception : ils emploient régulièrement des genres de discours dans leurs démonstrations, au point que certaines valeurs verbales sont dénommées par les genres de discours où elles apparaissent : présent de narration, présent sportif, infinitif de prescription, imparfait forain.... Ce qui n'arrive jamais en syntaxe : nulle « inversion du sujet didascalique » par exemple. C'est vraisemblablement la forte affinité générique des temps et modes verbaux qui explique cette prise en compte remarquable. 
Plus récemment toutefois, certains linguistes grammairiens se sont montrés sensibles à cette question, avec un succès certain dans la réception de leurs travaux. Nous pensons à Biber (1988 et 2009) et Blanche-Benveniste (1990). Cette popularité est en partie due à la méthodologie quantitative qui accompagnait ces travaux, en harmonie avec le succès actuel des théories basées sur les grand corpus, dynamisées par les progrès technologiques fulgurants et... le soutien des organismes officiels (ANR, AERES). Même si ce numéro n'est pas le lieu pour critiquer cette approche, l'article de Paul Cappeau, Françoise Gadet, Emmanuelle Guerin et Roberto Paternostro relève quelques problèmes liés au transfert vers l'informatique de compétences autrefois maîtrisées par le linguiste seul.

Biber explore deux directions. Grâce à l'informatique, il peut affiner la connaissance des éléments de langue qui soutiennent les genres. Ce type de travaux s'est largement répandu, avec un outil informatique sans cesse plus puissant (Malrieu et Rastier 2002), ou de façon artisanale, comme le montrent dans ce numéro André Petitjean et Claire Despierres lorsqu'ils traitent chacun d'un aspect linguistique du genre théâtral.

L'autre direction prise par Biber, à travers ce qu'il appelle «les dimensions », propose une articulation entre genre et grammaire. Les dimensions sont des groupes de faits - surreprésentés et/ou sous-représentés - dotés d'une fonction communicative pouvant caractériser des genres différents : les genres à haute densité d'information, les genres narratifs ou encore les genres à visée persuasive. On regrettera cependant que Biber parte de genres définis intuitivement, sans recul critique. Notre contribution, dans ce numéro, insistera sur la nécessité d'affiner la délimitation des genres étudiés.

Blanche-Benveniste (1999), en manipulant depuis longtemps des corpus conséquents, s'est rapidement aperçue que les genres discursifs n'étaient pas neutres. Elle a été conduite, en 1990, à avancer le principe qu'une grammaire seconde part du constat qu'à partir d'une grammaire première se greffent des faits de langue, inégalement maitrisés par les locuteurs, dont l'acquisition s'est faite suite à une introduction consciente par des locuteurs socialement actifs dans l'usage de la langue (grammairiens, journalistes, auteurs de manuels,...). Le but est toujours d'enrichir les moyens d'expression. Ces faits de langue (certains «en», «lequel» oblique, le style administratif,...) sont d'ailleurs souvent normativement marqués.

Les faits de langue considérés sont pris isolément et marquent, colorent le genre de discours plus qu'ils ne le construisent. Nous y voyons la limite principale de l'apport de la grammaire seconde : les faits concernés sont relativement limités.

L'approche «quantitative », numérisée ou non, n'est cependant pas la seule légitime. En effet, on associe parfois un peu trop vite «corpus attesté » et "genres de discours ». Pourtant, cette association ne se justifie pas plus que lorsqu'il s'agit d'études de syntaxe non générique. Les questions sans cesse débattues autour de l'attestation/ introspection/intuition relèvent du même type de réflexion. Les genres de discours ne 
sont pas des productions artificielles leur conférant un statut extérieur à l'intuition, comme pourrait l'être par exemple une langue inconnue. Il est vrai néanmoins que limiter l'étude grammaticale à un genre de discours entraine des difficultés supplémentaires. L'agrammaticalité correspond à une valeur relative, localisée dans un genre donné. La fréquence comparée d'un élément dans des genres différents est un paramètre utile. De plus, la capacité d'introspection du linguiste varie selon les genres de discours: pour des motifs socioculturels, les commentaires sportifs ou les horoscopes sont moins maitrisés que le théâtre ou les formulaires de déclarations d'impôts.

Enfin, les genres sont de tailles et d'accès très différents. Une étude quantitative des mises en garde inscrites sur les paquets de cigarettes ne serait guère sérieuse. Et il faudrait au moins dix ans pour obtenir, à force de persuasion (ou de fouilles illégales de sacs à main!) suffisamment d'agendas personnels pour prétendre à un corpus de taille incontestable.

Il ne s'agit pas de nier l'intérêt de ces travaux mais d'affirmer clairement que l'étude de la grammaire des genres de discours n'est pas davantage asservie par la technologie que l'étude de la grammaire de la langue. D'ailleurs, des approches qualitatives plus axées sur le fait commencent à émerger. Jean-Michel Adam, dans ce numéro, compare un même fait surreprésenté dans plusieurs genres de discours très différents et, ce faisant, contribue à préciser la valeur sémantique du fait indépendamment du genre du discours.

Dans un numéro précédent de LINX (2007), Simon Bouquet développe sa théorie : le fait est saisi dans toutes ses potentialités génériques dans le cadre méthodologique d'une levée d'homonymie. Simon Bouquet revient dans ce numéro examiner l'article premier de la déclaration des droits de l'homme. Dans le même cadre théorique, l'article de Créola Thénault s'emploie à décrire le présent de l'indicatif en fonction de ses propriétés génériques.

Cependant l'intérêt des grammairiens de profession pour les genres demeure très limité. Le désintérêt reste quasi général. Il suffit de lire les appels à communication, les actes de colloque pour constater combien les faits de langue ne sont jamais étudiés pour eux-mêmes à travers les genres de discours. Par refus ? Par désintérêt ? Le préjugé reste fort qu'une telle voie relève de l'exotisme ou de l'anecdote et non de la science. C'est d'autant plus curieux qu'il ne choque personne que de tels faits soient abordés par le biais d'un seul auteur - de préférence au programme de l'agrégation comme si l'auteur, fût-il génial, pouvait révéler à lui-seul les propriétés d'un fait de langue.

Il est peut-être une autre explication à ce désintérêt. En effet, la perception naturelle des genres n'est pas linguistique. Certes, les locuteurs distinguent facilement une recette de cuisine, un bulletin météo, une poésie ou un horoscope, mais la langue n'est pas immédiate dans la perception des GD. Ce que les locuteurs reconnaissent d'abord, voire exclusivement, c'est la face sociale, fonctionnelle, communicationnelle, culturelle de ces genres et non leur assise grammaticale. Un commentaire est d'abord « sportif » ou «mariage princier» mais le dispositif énonciatif mis en œuvre dans le 
« commentaire » n'est pas saillant. C'est pourquoi les classements de GD accordent le plus souvent la priorité à la perception sociale/culturelle des GD. Rastier (2001) hiérarchise les discours (littéraire, juridique), les champs génériques (théâtre, presse), les genres (comédie, tragédie, roman) et enfin les sous-genres (roman policier). Maigueneau (2004) part des discours constituants ou des hypergenres. Ces classements hiérarchiques contiennent leurs limites : les discours renvoient au moins autant à une division culturelle du monde qu'à des propriétés linguistiques précises, la différence entre genres et sous-genre reste poreuse et la tentation est forte d'imaginer des « soussous-genres ». Il n'en demeure pas moins que cette approche est plus intuitive et efficace qu'une stratification par la personne; par exemple le « je », qui rassemblerait et organiserait la poésie, la lettre de motivation, le journal intime.

Une preuve supplémentaire de l'impossibilité de classement par les faits de grammaire (Petitjean 1989) nous est fournie par les dénominations des genres de discours, indice fort que Branca (1999) perçoit très justement. Aucun élément terminologique linguistique n'y participe. On y observe des situations d'énonciation (entretien, commentaire, notice, recette, guide), des fonctions (montage, réclamation, programme, randonnée,...), des domaines d'activités (sport, littéraire) ou des supports médias (roman-photo, exposé oral, internet, TV). Généralement les catégories se combinent soit par composition (commentaire sportif, lettre de réclamation, notice de montage, entretien d'embauche, guide de randonnée, critique littéraire,...), soit par amalgame (courriel $=$ lettre par internet, pétition $=$ lettre collective de réclamation).

En revanche, aucun genre de discours ne contient dans sa dénomination la trace explicite d'un fait de langue (sauf peut-être les mots-fléchés), y compris quand cela pourrait se justifier empiriquement. Il n'y a pas par exemple de "commentaires sportifs au passé simple» (= commentaire sportif presse écrite) ou encore de « randonnée à l'infinitif de prescription » (guide de randonnée) qui s'opposerait à une « randonnée au passé simple ( (récit de voyage).

Tous les éléments grammaticaux sont-ils concernés par les genres de discours ? Assurément non. La langue utilisée par un genre de discours x est composée de trois sous-ensembles de faits grammaticaux :

- les faits qui ne sont jamais concernés par une sollicitation générique. Par exemple, l'ordre déterminant-nom : *banane la.

- les faits non sollicités par un genre mais susceptibles de l'être dans un autre. On pense au gérondif ( $S G A N A R E L L E$, en riant), caractéristique des didascalies mais transparent dans les commentaires sportifs. C'est également le cas des nominalisations déverbales, lesquelles, par la réduction qu'elles permettent du système des actants, sont particulièrement propices aux genres demandant concision et haute densité d'information (Biber 1988).

- les faits remarquables par leur absence ou leur surreprésentation. Par exemple, dans les commentaires sportifs, l'emploi prédicatif des noms propres, éventuellement avec une relative (Platini!, qui passe à Zidane !) 
La langue n'est pas la seule composante qui porte la «signification» communicationnelle culturelle ou sociale des genres. Certains paramètres influencent directement les formes employées : photos, encadrés, listes, médias, typographie,...

Ces éléments sont d'une importance variable selon les genres : l'ordonnance médicale est perçue, de loin, avant sa personnalisation. A l'inverse, la distinction entre une notice de montage et une recette de cuisine réside, si on excepte le lexique, dans la présence de schémas illustrant la procédure. Très peu dans la grammaire ${ }^{1}$.

Il ne faut pas omettre les facteurs non verbaux pour les genres oraux (Kerbrat et Traverso, 2004), genres dont la délimitation est plus difficile à établir, peut-être à cause de leur protocole moins construit que les genres écrits. On trouvera ici une contribution de Florence Lefeuvre sur les particules discursives bon et quoi.

Ces paramètres non linguistiques participent à la construction de faits de langue (Martin et Rose, 2010). C'est même une caractéristique forte des relations entre genres et grammaire. Illustrons cela par les absences de sujets fréquemment constatées sur les emballages (se boit très frais), les petites annonces (vends Xanthia 1996) ou les carnets de note (doit davantage travailler) dont Salima El Hadi fait ici une description associant marques linguistiques et fonction sociale. Le support matériel cadre l'identification sémantique du « vide » observé en position sujet. Reste alors au grammairien à justifier cette intégration (ou ces intégrations) du non linguistique dans des positions syntaxiques, intégration relativement aisée quand il s'agit du sujet, mais guère envisageable quand il s'agit d'un complément d'objet, sauf à l'infinitif (« ne pas utiliser » inscrit sur le sèche-linge d'une Lavomatic).

Un regard grammatical sur les genres de discours fait apparaître des macrostructures qu'il est difficile de classer dans une typologie en tant qu'unités autonomes, bien que ces macrostructures soient reconnaissables par les locuteurs. Considérons à cette fin les titres. Ils ne sont généralement pas tenus pour des genres de discours. A la limite, une discussion peut s'engager sur certains titres typés, appréhendables de façon autonome relativement à l'objet qu'ils ont pour mission première d'étiqueter (par exemple les titres du journal «Libération» ou du «Canard Enchainé »). Pourtant, le versant grammatical est aisé à repérer car les titres abondent de particularités syntaxiques qu'ils ne partagent pas toujours avec d'autres genres de discours. C'est vrai des interrogatives indirectes indépendantes, exemplifiées cidessous. Ces propositions se réalisent librement, sans dépendre d'un terme régissant. A notre connaissance, seuls les titres hébergent cette structure.

A quoi sert notre inconscient (psychologies mars 2011)

Où est la policière la plus courageuse du monde (Aujourd'hui 6/3/2011)

\footnotetext{
${ }^{1}$ La recette de cuisine est cependant moins contrainte au sens de Maingueneau (2004), ne serait-ce que parce qu'elle peut être signée.
} 
Aussi nous trouvons-nous devant une difficulté. Certes, les titres ont un ensemble cohérent de singularités grammaticales qui les rapprochent formellement des GD. Toutefois, ils n'ont pas l'autonomie socio-culturo-communicationnelle qu'on attend des genres ou sous-genres de discours. Malgré cela, pour l'étude grammaticale, les titres sont (presque) autant des «genres» que les recettes de cuisine : le locuteur reconnaitra dans les deux cas une macrostructure grâce à ses spécificités grammaticales. Aussi proposons-nous d'intégrer les titres dans une catégorie que nous appellerons «genre méronyme $»^{2}$. En effet, s'ils ont l'autonomie suffisante qui les identifie grammaticalement, les titres participent à la composition de nombreux genres écrits. En outre, comme l'indique Maingueneau (2012), le titre a des relations linguistiques avec le reste du genre. Autrement dit, les genres méronymes ne sont pas insérés de façon étanche dans le genre qui les appelle.

Les genres méronymes sont nombreux : les légendes, les listes d'ingrédients, les en-têtes ou les didascalies. Dans ce numéro, Julie Lefebvre traitera des notes de bas de page.

Les genres méronymes ne doivent toutefois pas être confondus avec l'insertion d'un genre dans un autre, par exemple pour constituer des scénographies particulières. Sylvie Freyermuth étudiera ici une insertion de recette de cuisine dans un passage d'un roman de Jean Rouaud.

Il reste encore bien d'autres questions soulevées par la relation entre la grammaire et les genres de discours, qui trouveront des échos dans l'ensemble des contributions réunies dans ce numéro de LINX, ainsi que dans Despierres et Krazem (2012a) :

- Les genres de discours créent-ils de la «néologie grammaticale »?

- Comment intégrer les variations grammaticales individuelles ou collectives?

- Pourquoi certains éléments grammaticaux sont-ils susceptibles d'être marqués génériquement mais pas d'autres?

- Quelle est la place des genres de discours dans l'ensemble du système linguistique?

Autant de questions théoriques dont les enjeux ne laisseront pas indifférents tous ceux qui accordent quelque crédit à l'intuition de Bakhtine (ou de son cercle), associant genres de discours et acquisition de la langue.

\footnotetext{
2 Rastier (2001) parle de «genres inclus ». Nous préférons le terme «méronyme » car il saisit une propriété relationnelle.
} 
Mustapha Krazem

\section{BIBLIOGRAPHIE}

BAKHTine, M., 1984, «Les genres de discours » dans Esthétique de la création verbale, Paris, Gallimard.

BIBER D., 1988, Variation across speech and writing, Cambridge University Press.

Biber D., et COnRaD, S., 2009, Register, Genre, and Style, New York, Cambridge University Press.

BlanCHE-BENVENISTE Cl., 1990, «Grammaire première et grammaire seconde, l'exemple de en », Recherches sur le français parlé, n 10 , PU de Provence, p. 96-110.

BlanChE-BenVEniste, Cl., 1999 "Constitution et exploitation d'un grand corpus », RFLA volume IV fascicule 1, p. 65-74.

BRANCA, S., 1999, «Types, modes et genres : entre langue et discours », Langage et Société n87.

BONNARD, H., 1982, Code du français courant, Paris, Magnard.

BOUQUET, S., 2007, «Contribution à une linguistique néo-saussurienne des genres de la parole : une grammaire du morphème $\mathrm{ON} », \operatorname{Linx} \mathrm{n}^{\circ} 56$, Paris, Université de Paris XII Nanterre, p. 143-156.

DoLININE, C., 1999, «Le problème des genres du discours quarante-cinq ans après Bakhtine », Langage et Société $\mathrm{n}^{\circ} 87$, p. 25-40.

Despierres, Cl. et KraZEM, M., 2012b (eds), Quand les genres de discours provoquent la grammaire et réciproquement, Lambert Lucas.

Despierres, Cl. et Krazem, M., 2012b « De l'exemple en grammaire des genres de discours » dans Quand les genres de discours provoquent la grammaire et réciproquement, Lambert Lucas, p. 67-76.

Kerbrat, C. et Traverso, V., 2004, «Types d'interactions et genres de l'oral », Langages, nº153, p. 41-51..

LE GOFFIC, P., 1993, Grammaire de la phrase française, Paris, Hachette.

Maingueneau D., 2004, « retour sur une catégorie le genre » dans Adam J.M, Grize J.B et Ali Bouacha M., Texte et discours : catégorie pour l'analyse EUD Dijon, p. 107-118.

MaingueneaU, D., 2012, Les phrases sans texte, Armand Colin.

MALRIEUX, D. et Rastier, F., 2001, « Genres et variations morphosyntaxiques », T.A.L, vol. 42, $\mathrm{n}^{\circ} 2$, p. $548-577$.

Martin, J et Rose, D., 2008, Genre relations. Mapping culture, London, Equinox.

Petitjean, A., 1989, « Les typologies textuelles », Pratiques, 62, Metz, p. 86-125.

Sancier ChÂteau, A., Denis, D., 1997, Grammaire du français, Paris, Le Livre de poche.

Rastier, F., 2001, Arts et sciences du texte, Paris, PUF. 